\title{
ON A CERTAIN METRIC ASPECT OF PLANE PROJECTIVE TRANSFORMATIONS*
}

\section{BY LULU HOFMANN}

1. Introduction and Results. This paper contains the study of a special type of metric relations between projective planes. To bring out clearly the point of view from which the planes are considered, we first briefly summarize the principal known facts about the subject in general, remarking that by "metric" relations we refer merely to properties based on the ordinary euclidean measure of a straight line segment and an angle.

Let $\pi$ and $\pi^{\prime}$ be two projectively related planes. $\dagger$ At a general point of $\pi\left(\pi^{\prime}\right)$ there are $\infty^{1}$ angles and on a general line $\infty^{1}$ segments which preserve their size under the transformation. These angles and these segments form an involution at the point and on the line.

There exist, however, in $\pi$ and $\pi^{\prime}$ two distinguished pairs of corresponding points, the focal points, and two distinguished pairs of corresponding lines, the equi-segmental axes, such that all corresponding angles at these points and all corresponding segments on these lines are congruent. In either plane, these points and lines lie symmetrically with regard to the vanishing line. If the distance of the focal points from the vanishing line is $c$ in $\pi$ and $c^{\prime}$ in $\pi^{\prime}$, then the distance of the equi-segmental axes from this line is $c^{\prime}$ in $\pi$ and $c$ in $\pi^{\prime}$. The quantities $c$ and $c^{\prime}$ are called the parameters of the transformation, and the line joining the focal points in either plane is called the focal axis.

Thus in two projective planes, the metric properties of

* Presented to the Society, October 27, 1928.

$\dagger$ For the following see H. J. S. Smith, Memoir on the focal properties of homographic figures, Collected Works, vol. 1, p. 545-602. We assume with Smith throughout this paper that the projective transformation considered is a general one, and we exclude the affine case. 
corresponding segments on corresponding lines and of corresponding angles at corresponding points are known. In this paper, we start out from a new combination of elements, namely we study the metric properties of corresponding segments at corresponding points. We choose an arbitrary pair of corresponding points $P_{0}$ and $P_{0}^{\prime}$ in $\pi$ and $\pi^{\prime}$ and examine those segments radiating out from $P_{0}$ which preserve their length as they are transformed into segments radiating out from $P_{0}^{\prime}$. In other words, after choosing a fixed pair of corresponding points $P_{0}$ and $P_{0}^{\prime}$, we determine the locus of the points $P$ of $\pi$ such that the segment $P P_{0}$ is congruent to the transformed segment $P^{\prime} P_{0}^{\prime}$.

The locus of the points $P$ of $\pi$ such that

$$
P P_{0}=P^{\prime} P_{0}^{\prime}
$$

where $P_{0}$ is fixed, is found to be a bipartite quartic $Q$ of genus one. Its two branches* lie on opposite sides of the vanishing line; they intersect only at the infinite point of this line, thus making it a double point, and each there has the corresponding equi-segmental axis for its asymptote.

The second double point of $Q$ lies at the fixed point $P_{0}$. It is a node, a cusp, or an isolated double point, according as there are two, one, or no real line elements at $P_{0}$ which are equal to their corresponding elements at $P_{0}^{\prime}$. The points of $\pi$ belonging to the second class form a bipartite sextic which separates those of the first from those of the third class.

The quartic $Q$ is tangent to the circles about $P_{0}$ as center at the cyclic points. On any such circle there are therefore four, and on a general algebraic curve of the $n$th order $\dagger 4 n$ finite points obeying condition (1).

* For the sense in which we use the word branch we refer, for example, to the definition of complete branch given by H. G. Zeuthen in the first paragraph of his paper Sur les differrentes formes des courbes planes $d u$ quatrieme ordre, Mathematische Annalen, vol. 7 (1874), pp. 420-432. In translation: "We denote by complete branch the part of a plane curve which can be described in a continuous manner by a point which, after having passed once through every fixed point, returns to its initial position."

$\dagger$ A curve not passing through the cyclic points and the infinite point of the vanishing line. 
Aside from all metric considerations, $Q$ may be described in a purely projective manner as the locus of the $\infty^{1}$ double points determined by the projectivity of $\pi$ and $\pi^{\prime}$, as these planes are laid onto one another in the $\infty^{1}$ ways possible such that $P_{0}$ and $P_{0}{ }^{\prime}$ coincide.

The extension to the case where corresponding segments at corresponding fixed points are required not to be equal but to form an arbitrary fixed ratio $k>0$, so that $P P_{0}=k \cdot P^{\prime} P_{0}^{\prime}$, furnishes nothing new. It is identical with the original problem for the transformation with the parameters $c$ and $\sqrt{ } k \cdot c^{\prime}$.

2. Fundamental Properties of the Locus $Q$, its Double and Infinite Points, its Real Asymptotes, and its Branches. We represent the projective relation between $\pi$ and $\pi^{\prime}$ in the canonical form,* choosing the focal axis and the vanishing line in either plane as the $x y$ and $x^{\prime} y^{\prime}$ axes. The transformation formulas then are

$$
x^{\prime}=\frac{c c^{\prime}}{x}, \quad y^{\prime}=\frac{c^{\prime} y}{x} ;
$$

$c$ and $c^{\prime}$ are the parameters of the transformation; the focal points of $\pi\left[\pi^{\prime}\right]$ have the coordinates $( \pm c, 0)\left[\left( \pm c^{\prime}, 0\right)\right]$ and the equi-segmental axes the equations $x= \pm c^{\prime}\left[x^{\prime}= \pm c\right]$.

Let $P_{0}\left(x_{0}, y_{0}\right)$ and $P_{0}^{\prime}\left(x_{0}^{\prime}, y_{0}^{\prime}\right)$ be the fixed pair of corresponding points. Then the locus of the points $P$ such that

$$
P P_{0}=P^{\prime} P_{0}^{\prime}
$$

is obtained by replacing in

$$
\left(x-x_{0}\right)^{2}+\left(y-y_{0}\right)^{2}=\left(x^{\prime}-x_{0}^{\prime}\right)^{2}+\left(y^{\prime}-y_{0}^{\prime}\right)^{2}
$$

the primed coordinates by the unprimed ones according to the formulas (2) of transformation. After ridding the resulting expression of its denominators and writing it in the homogeneous coordinates $x, y, t$, instead of $x, y$, we obtain

(3) $x_{0}{ }^{2} x^{2}\left[\left(x-x_{0} t\right)^{2}+\left(y-y_{0} t\right)^{2}\right]$

$$
-c^{\prime 2} t^{2}\left[c^{2}\left(x-x_{0} t\right)^{2}+\left(x y_{0}-y x_{0}\right)^{2}\right]=0 .
$$

* See the above cited paper by Smith, p. 561. 
The locus (3) of the points $P$ fulfilling condition (2) is a quartic curve. We call it $Q$. It is obvious from (3) that $Q$ has double points at $\left(x_{0}, y_{0}\right)$ and $(x=t=0)$, that is, at the fixed point $P_{0}$, and at the infinite point of the vanishing line (y-axis).

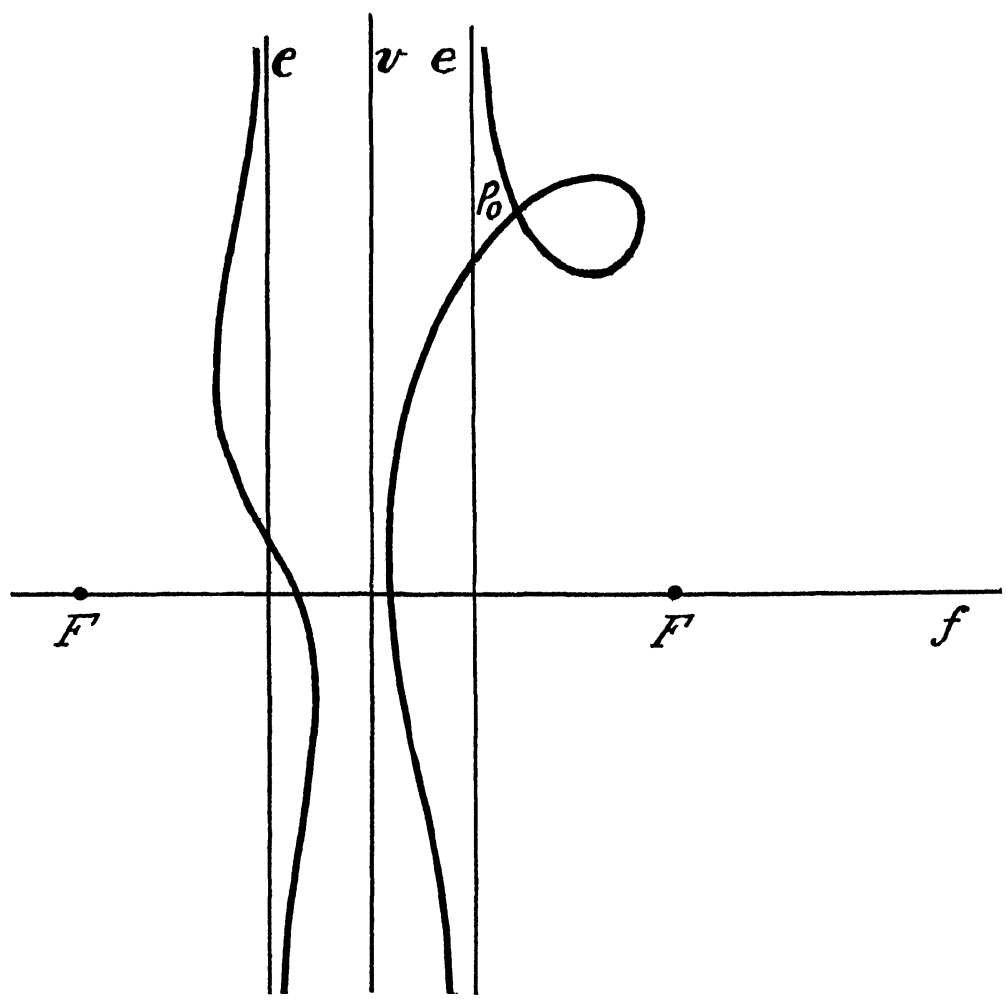

Fig. 1. Schematic Figure of $Q$ (Focal points $F$; focal axis $f$; vanishing line $v$; and equi-segmental axes $e$ ).

The substitution of $x= \pm c^{\prime} t$ into (3) furnishes two expressions with the factor $t^{3}$. It follows that $Q$ is tangent to the two equi-segmental axes at infinity and therefore always has a node at the infinite point of the vanishing line.

Besides this node, $Q$ has in common with the vanishing line the points $(0, \pm i c)^{*}$ and with the line at infinity the

* These points correspond to the cyclic points of $\pi^{\prime}$. 
cyclic points. Thus, since it does not have any real finite points on the vanishing line, nor any real infinite points except $(x=t=0)$, and since it has two real distinct asymptotes, parallel to and lying on opposite sides of the vanishing line, it follows that $Q$ consists of two branches separated by the vanishing line and intersecting only at the infinite point of this line. Obviously the branches are odd (they have only one point of intersection).

If we consider a segment $P P_{0}$ not only with regard to its end points but with regard to all the points which make it up, we have to distinguish between the two branches of $Q$. For a segment with its end point $P$ on that branch of $Q$ which lies on the same side of the vanishing line as $P_{0}$, is transformed point by point to the segment $P^{\prime} P_{0}^{\prime}$; while, if $P$ lies on the other branch, the segment $P P_{0}$ contains a point of the vanishing line and corresponds therefore point by point not to $P^{\prime} P_{0}^{\prime}$ itself but to the complementary infinite segment.

3. The Intersections of $Q$ with the Circles about $P_{0}$ and the Character of this Point as a Double Point. The Sextic S. Properties of Symmetry of Plane Projectivities. It is obvious from the first term of (3) that $Q$ not only contains the cyclic points but is there tangent to the circles about $P_{0}$ as center. It follows that $Q$ has in common with any such circle four finite points; when the radius of the circle is sufficiently large, they are all real, as is evident from the character of $Q$. There is only one imaginary circle which intersects $Q$ three times at each of the cyclic points.

If $r$ is an arbitrary length, there are always exactly four points at this distance from $P_{0}$ which are transformed into points at the same distance from $P_{0}^{\prime}$.

To find the four points of $\pi$ belonging to a certain distance $r$, independently of $Q$, we invert for a moment the hitherto considered order of transformation, regarding $\pi^{\prime}$ as the original and $\pi$ as the transformed plane. A circle of radius $r$ about $P_{0}^{\prime}$ is transformed into a certain conic of $\pi$. The four points in question are found by intersecting this conic with the circle of radius $r$ about $P_{0}$ itself. 
If we take for $r$ a vanishing quantity $d r$, the conic in $\pi$ becomes a vanishing ellipse with $P_{0}$ as a center* and therefore has in common with the concentric circle of radius $d r$ two real distinct, coincident, or imaginary diameters. This means there are two real distinct, coincident, or imaginary line elements at $P_{0}$ which are equal to the corresponding elements at $P_{0}^{\prime}$, and accordingly $P_{0}$ is a node, a cusp, or an isolated double point for $Q$. We denote the points of the three different types as $N$-, $C$-, and $I$-points.

We determine the character of $P_{0}$ analytically, by demand. ing that it be a triple point of intersection with $Q$ for the line $y-y_{0}=\alpha\left(x-x_{0}\right)$ and investigating when, under this condition, $\alpha$ assumes two real distinct, coincident, or imaginary values. Calculation shows that these cases occur according as

$$
c^{\prime 2} x_{0}{ }^{4} t_{0}^{2}+c^{\prime 2} x_{0}^{2} t_{0}^{2}\left(y_{0}^{2}+c^{2} t_{0}^{2}\right)-x_{0}^{6}-c^{2} c^{\prime 4} t_{0}{ }^{6}
$$

is greater than, equal to, or less than zero.

Expression (4) equated to zero and considered as an equation in $x_{0}, y_{0}, t_{0} \dagger$ shall be referred to as the sextic $S . S$ is the locus of the $C$-points and separates the $N$-points from the $I$-points.

For the intersections of $S$ with the focal axis we have $x_{0}= \pm c^{\prime}, \pm\left(c c^{\prime}\right)^{1 / 2}, \pm i\left(c c^{\prime}\right)^{1 / 2}$. Let us denote by $\Gamma$ and $\gamma$ the greater and smaller of the quantities $c$ and $c^{\prime}$. It is easily seen that both when $\left|x_{0}\right|<\gamma$ and when $\left|x_{0}\right|>\Gamma,(4)$ is negative, while it is positive when $\left|x_{0}\right|=c^{\prime}, y_{0} \neq 0$. This indicates the division of $\pi$ into the regions $N$ and $I$ as shown in Fig. 2 .

We easily deduce the main properties of $S$ by equating (4) to zero. $S$ is symmetric with regard to the vanishing line and the focal axis. At the infinite point of the vanishing line, it has four coinciding points of intersection with a general line, and six with the vanishing line itself and with

* Disregarding infinitesimals of higher order than $d r$. See Smith, p. 559.

$\dagger$ We have again replaced the non-homogeneous coordinates by homogeneous ones. The suffix zero merely indicates that the respective point appears in the character of the fixed point of our problem. 
the focal axis. Thus these two lines are the only tangents of $S$ at its quadruple infinite point. Referring to the symmetric character of the curve, one can show that this point, besides being the only infinite one is also the only multiple one of $S$. It follows that $S$ consists of two branches which each have a point of self contact at the infinite point of the vanishing line. The first branch intersects the focal axis at the points $x= \pm \gamma$ and is tangent at infinity to the vanishing line. The second intersects the focal axis at the points $x= \pm \Gamma$ and is tangent at infinity to the line at infinity itself.

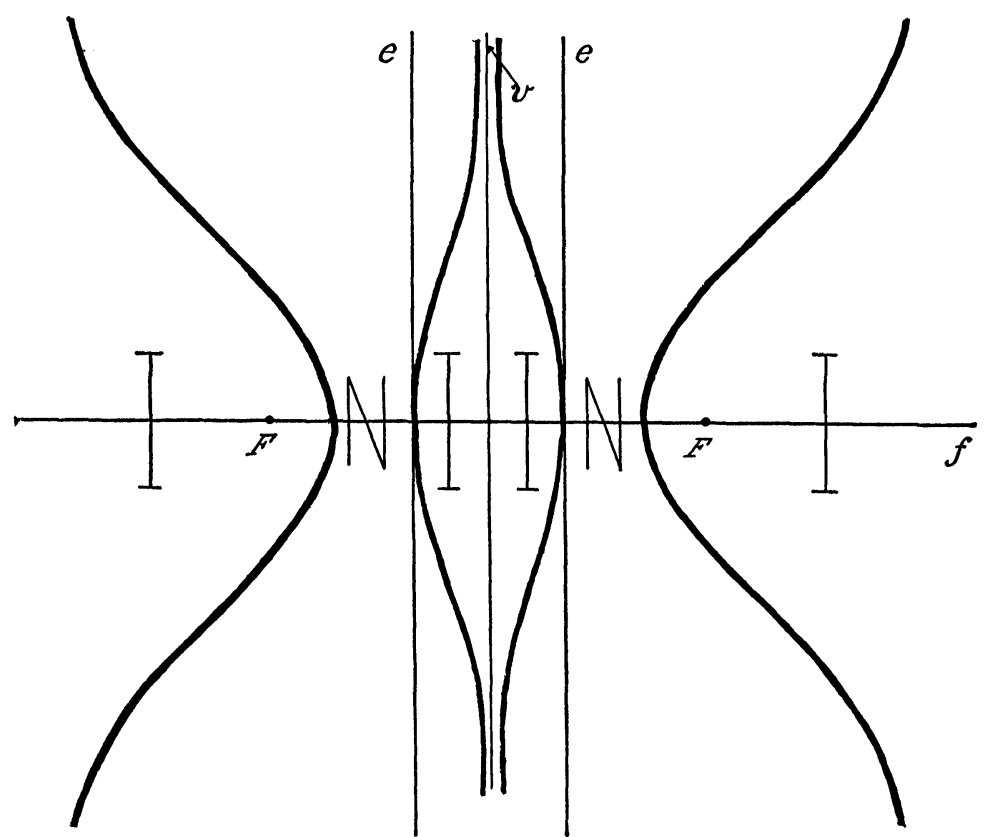

Fig. 2. Schematic Figure of $S$.

We have stressed the symmetric character of $S$. We add the remark that two curves $Q^{(1)}$ and $Q^{(2)}$ belonging to points $P_{0}{ }^{(1)}$ and $P_{0}{ }^{(2)}$ which lie symmetrically with regard to the vanishing line or the focal axis, are mutually symmetric with regard to the respective line. This includes as a special statement that the $Q$-curve belonging to a point $P_{0}$ on the focal 
axis, is itself symmetric with regard to this axis. The metric point of view brings out the symmetric properties of plane projectivities.

4. Interpretation of $Q$ as $\pi$ and $\pi^{\prime}$ Coincide and Proof that it is of Genus One. Let $\pi^{\prime}$ be laid on $\pi$ in such a manner that $P_{0}$ and $P_{0}^{\prime}$ coincide. There will be two more points, say $P_{1}$ and $P_{2}$ of $\pi$ which coincide with the corresponding points of $\pi^{\prime}$, thus with $P_{0}$ forming the three double points of the transformation. By definition, $P_{1}$ and $P_{2}$ lie on $Q$; for, being a double point of the transformation, $P_{1}=P_{1}^{\prime}$ $\left(P_{2}=P_{2}^{\prime}\right)$ as a point of $\pi$ has the same distance from $P_{0}$ that it has from $P_{0}^{\prime}$ as a point of $\pi^{\prime}$. Thus, $Q$ is the locus of the $\infty^{1}$ double points determined by the projectivity as $\pi^{\prime}$ is laid on $\pi$ in the $\infty^{1}$ ways possible such that $P_{0}$ and $P_{0}^{\prime}$ coincide.

Choosing one definite such position of $\pi$ and $\pi^{\prime}$ with the double points $P_{0}, P_{1}$ and $P_{2}$, let us consider the line $l$ through $P_{0}$ and $P_{1}$. It cuts $Q$ in a fourth point ( $P_{0}$ counting as two), say $P_{1} *$. The point $P_{1}$ * belongs, as a double point of the transformation, to that position of $\pi$ and $\pi^{\prime}$ which is obtained from the one considered by rotating $\pi^{\prime}$ through an angle of $180^{\circ}$ about the perpendicular to $l$ which lies in $\pi$ and passes through $P_{0}$. Accordingly, $P_{1}$ and $P_{1} *$ can coincide only at the point $P_{0}$ or at infinity. On the other hand, the coincidence of $P_{1}$ and $P_{1}^{*}$ is equivalent with the existence of a double point of $Q$, so that double points of $Q$ can occur only at $P_{0}$ and at infinity. But we know that $Q$ has only one infinite double point, namely, at $x=t=0$. Thus we have proved that $Q$ has exactly two double points and is therefore of genus one.

5. Degenerations of $Q$ and Specializations of $S$. To study the cases in which $Q$ degenerates, we let $P_{0}$ assume all possible positions excepting those on the vanishing line and the line at infinity. The character of our problem makes it natural to consider only such finite points $P_{0}$ which are again transformed into finite points $P_{0}^{\prime}$.

Owing to the fact that $Q$ consists of two branches lying on opposite sides of the vanishing line and each having one of 
the equi-segmental axes for its asymptote, the only possible degenerations of the curve are those in which it contains one or both of these axes as a part. Calculation shows that in the first case $P_{0}$ lies on one of the equi-segmental axes $\left(x_{0}=(-) c^{\prime}\right)$ and in the second case at one of the focal points $\left(x_{0}={ }_{(-)}^{+} c, y_{0}=0\right)$. The corresponding equations for $Q$ are

$$
\begin{gathered}
\left(x(\mp) c^{\prime} t\right)\left[\left(x(\mp) c^{\prime} t\right)\left(x^{2}-c^{2} t^{2}\right)+y\left\{x\left(y-2 y_{0} t\right)\left( \pm c^{\prime} y t\right\}\right]=0,\right. \\
\left(x^{2}-c^{\prime 2} t^{2}\right)\left[(x(\mp) c t)^{2}+y^{2}\right]=0 .
\end{gathered}
$$

When $P_{0}$ lies on one of the equi-segmental axes, $Q$ consists of the respective axis and a cubic, which is made up of an oval through $P_{0}$ and an odd branch with the other axis for its asymptote. The branches lie on opposite sides of the vanishing line and contain the respective focal points.

When $P_{0}$ lies at one of the focal points, $Q$ consists of the absolute lines through this point and both equi-segmental axes.

The degenerations of $Q$ are caused by special positions of $P_{0}$ and occur in every projectivity, that is for every value of the parameters $c$ and $c^{\prime}$. S, however, depends on these parameters only. It is evident from its constitution that it cannot degenerate, but that it specializes when $c=c^{\prime}$. In this case it becomes unipartite and adds to its quadruple singularity at infinity two nodes at the focal points. On the contrary, the equality of $c$ and $c^{\prime}$ has no essential effect on a general $Q$-curve.

6. Extension of the Problem to the Case of Corresponding Segments forming a General Fixed Ratio $k>0$. We have so far investigated the locus of those points of $\pi$ whose distances from a fixed point $P_{0}$ remain unchanged under the transformation. The investigation immediately extends itself to the case in which we require these distances to be changed according to any fixed ratio $k(k>0): P P_{0}=k \cdot P^{\prime} P_{0}^{\prime}$. Then (3) assumes the form

(3) $\quad x_{0}^{2} x^{2}\left[\left(x-x_{0} t\right)^{2}+\left(y-y_{0} t\right)^{2}\right]$

$$
-k c^{2} t^{2}\left[c^{2}\left(x-x_{0} t\right)^{2}+\left(x y_{0}-y x_{0}\right)^{2}\right]=0 \text {. }
$$


But this is the equation corresponding to the original problem $(k=1)$ for a projectivity with the parameters $c$ and $\sqrt{ } k \cdot c^{\prime}$. The new curves $Q_{k}$ and $S_{k}$ are therefore of exactly the same type as $Q$ and $S$. The $\infty^{1}$ curves $Q_{k}$ belonging to the same point $P_{0}$ form a pencil whose base points are $P_{0}$ itself and the infinite point of the vanishing line, each counted four times, and the cyclic points and the points $(0, \pm i c)$, each counted twice.

7. Summary. In conclusion, we repeat the fundamental results of the preceding discussion in a form no longer distinguishing $\pi$ before $\pi^{\prime}$, but stressing the obvious symmetry of the problem with regard to both planes.

In two projective planes there is associated with every fixed pair of corresponding points and every value of a positive quantity $k$ a pair of bipartite quartics of genus one corresponding in the projectivity which are the loci of all the pairs of corresponding points whose distances from the respective fixed points form the ratio $k$. Moreover there is associated with every value of $k$ a pair of bipartite sextics corresponding in the projectivity which are the loci of all those pairs of corresponding points at which there exists one real pair of corresponding line elements forming the ratio $k$. These sextics separate the points of their planes where there are two such pairs from the points where there is none.

The problem discussed in this paper immediately suggests two new ones. First, the problem corresponding by duality: the study of the pairs of corresponding lines forming congruent angles with two corresponding fixed lines. Secondly, the extension to spaces of higher dimensions. These problems lead to curves of the fourth class and hypersurfaces of the fourth order, but do not appear to furnish essentially new results.

Columbia University 